\title{
Gamma-ray absorption in the microquasar SS433
}

\author{
M. M. Reynoso ${ }^{1,2}$ \\ Departamento de Física, Facultad de Ciencias Exactas y Naturales \\ Universidad Nacional de Mar del Plata, Funes 3350, (7600) Mar del Plata, Argentina \\ H. R. Christiansen \\ State Univesity of Ceará, Physics Dept., Av. Paranjana 1700, 60740-000 Fortaleza - CE, Brazil \\ G. E. Romero ${ }^{3}$ \\ Instituto Argentino de Radioastronomía, CONICET, C.C.5, (1894) Villa Elisa, Buenos Aires, Argentina and Facultad de Ciencias \\ Astronómicas y Geofísicas, Universidad Nacional de La Plata, Paseo del Bosque, 1900 La Plata, Argentina
}

\begin{abstract}
We discuss the gamma-ray absorption in the inner region of the microquasar SS433. Our investigation includes several contributions to the opacity of this system. They result from the ambient fields generated by the primary star, possibly an A-type supergiant, and a very extended disk around the black hole. Besides the sharp and dramatic absorption effect that occurs every time the star crosses the emission zone, we find in the UV photon field from the extended disk an important source of absorption for very high energy gamma-rays. This results in periodic gamma-ray observational signatures.
\end{abstract}

"Key words: gamma-rays: theory, X-rays: binaries, radiation mechanisms: non-thermal, stars: winds, outflows

\section{INTRODUCTION}

SS433 is a particularly interesting X-ray binary system that consists of a donor star feeding mass to a black hole [1] in orbit with a period of $13 \mathrm{~d}$. From the vicinity of the compact object two oppositely directed jets are launched developing regular precession with a period of $162 \mathrm{~d}$. These jets can be considered as 'dark' [2] because their main power output is given by their kinetic luminosity, $L_{\mathrm{k}} \sim 10^{39} \mathrm{erg}$ $\mathrm{s}^{-1}$. Indeed, the ejected matter has determined the deformation of the nebula W50 that surrounds the SS433 system [3].

Gamma-ray emission from similar objects has been confirmed very recently $[4,5,6]$, so the study of absorption in the complex case of SS433 is important to characterize the possible high energy emission that this source might present to instruments such as GLAST and the new Cherenkov arrays MAGIC II and VERITAS. For a recent discussion of the absorption in the case of other systems like LS I +61 303 and LS 5039, see for instance, Ref. [7,8,9].

\footnotetext{
1 E-mail address: mreynoso@mdp.edu.ar (M.M. Reynoso).

2 Fellow of CONICET, Argentina

3 Member of CONICET, Argentina
}

Regardless of any specific gamma-ray emission process that could operate in SS433, in the present work, we shall focus on the possible sources of gamma-ray absorption taking place mainly in the inner region and in the immediate vicinity of the binary. There, the interactions with photons and matter can be important and cause significant absorption. In particular, if the emission is originated near the compact object, we find a dramatic absorption effect occurring every time the companion star eclipses the emission zone. Furthermore, at very high energies $\left(E_{\gamma} \gtrsim 50\right.$ $\mathrm{GeV}$ ), we predict a very important absorption effect caused mainly by the UV emission from the extended disk around the accretion disk, and secondly by the mid-IR emission from the same disk.

We have based our work on the set of parameters that are currently believed to describe the system SS433 after more that 20 years of observation and study. These parameters are summarized in the next section. The resulting absorption signatures are presented in Sect. 3, and a discussion is left for Sect. 4, where we analyze the possible detection of the specific absorption features. 


\section{THE SOURCE}

The mass loss rate in the jets of SS433 is $\dot{m}_{\mathrm{j}}=5 \times$ $10^{-7} M_{\odot} \mathrm{yr}^{-1}$ and their bulk velocity is $v_{\mathrm{b}} \approx 0.26 \mathrm{c}$. The normal to the orbital plane makes an angle $\theta \approx 21^{\circ}$ with the approaching jet and an angle $i=78^{\circ}$ with the line of sight. The line of sight then makes a angle $i_{\mathrm{j}}$ with the jet which is time-dependent because of precession (see Fig.1).

A thick expanding disk wind believed to be fed by the supercritical accretion encloses also the star [10]. According to Ref. [1], the disk wind has a half opening angle $\alpha_{\mathrm{w}} \approx 30^{\circ}$, a mass loss rate $\dot{M}_{\mathrm{w}} \approx 10^{-4} M_{\odot} \mathrm{yr}^{-1}$, and a velocity $v_{\mathrm{w}} \sim$ $1500 \mathrm{~km} \mathrm{~s}^{-1}$. Emission of UV photons from this extended disk will be considered using a blackbody distribution with $T_{\mathrm{UV}}=21000 \mathrm{~K}$ following Ref. [11], while mid-IR emission will be taken into account using a fit for the flux density presented in Ref. [12].

The spectral identification of the primary star has been difficult because of the presence of the extended disk, since the star is often partially or totally obscured by it. After convenient observations at specific configurations of precessional and orbital phases it has become quite clear that the star is an A-supergiant $[13,14,15]$. We assume the mass of the components as derived from INTEGRAL observations [13], $M_{\mathrm{bh}}=9 M_{\odot}$ and $M_{\star}=30 M_{\odot}$ for the black hole and the star respectively, which correspond to an orbital separation $a \simeq 79 R_{\odot}$ for a zero-eccentricity orbit as is the case for SS433. Since the star is believed to fill its Roche lobe, the implied radius according to Ref. [16] is $R_{\star}=$ $0.49 q^{-2 / 3} a\left[0.6 q^{-2 / 3}+\ln \left(1+q^{-1 / 3}\right)\right]^{-1} \simeq 38 R_{\odot}$, where $q=M_{\mathrm{bh}} / M_{\star}$. We shall consider the emission of soft photons from the star through an associated blackbody distribution with an effective temperature $T_{\star}=8500 \mathrm{~K}[14,15]$.

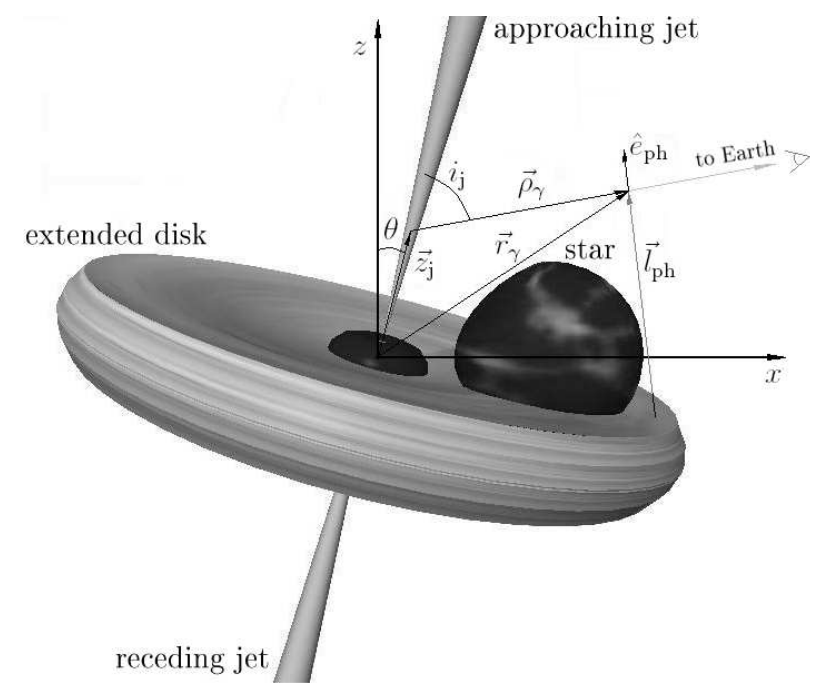

Fig. 1. Schematic view of SS433. The approaching jet is most of the time closer to the line of sight and the receding one is oppositely directed.

\section{OPACITY TO GAMMA-RAY PROPAGATION}

Attenuation of the putative gamma radiation emitted by this source would be mainly due to $e^{-} e^{+}$pair production arising from $\gamma \gamma$ annihilation, particularly with lower energy photons coming from the extended disk and from the star. Matter, on the other hand, also offers a significant target for gamma-rays emitted by the system. We shall calculate also the effect of $\gamma N$ interactions around the star and the extended disk.

When a gamma-ray of energy $E_{\gamma}$ travels a distance $d \rho_{\gamma}$ in a photon field, there is a differential optical depth associated with it. Absorption correspondingly arises from the gamma-ray interaction with soft photons of energy $E$. Assuming that the former runs following $\hat{e}_{\gamma}$ and the latter are directed along $\hat{e}_{\mathrm{ph}}=\left(\sin \theta^{\prime} \cos \phi^{\prime}, \sin \theta^{\prime} \sin \phi^{\prime}, \cos \theta^{\prime}\right)$, the corresponding optical depth is given by [17],

$d \tau_{\gamma \gamma}=\left(1-\hat{e}_{\gamma} \cdot \hat{e}_{\mathrm{ph}}\right) n_{\mathrm{ph}} \sigma_{\gamma \gamma} d \rho_{\gamma} d E d \cos \theta^{\prime} d \phi^{\prime}$.

Here, $n_{\mathrm{ph}}$ is the density of the soft photons per solid angle and soft photon energy units. The angles $\theta^{\prime}$ and $\phi^{\prime}$ are taken in a convenient coordinate system and the cross section for the process $\gamma \gamma \rightarrow e^{+} e^{-}$is given by

$$
\begin{array}{r}
\sigma_{\gamma \gamma}\left(E_{\gamma}, E\right)=\frac{\pi r_{0}^{2}}{2}\left(1-\xi^{2}\right) \times \\
{\left[2 \xi\left(\xi^{2}-2\right)+\left(3-\xi^{4}\right) \ln \left(\frac{1+\xi}{1-\xi}\right)\right],}
\end{array}
$$

where $r_{0}$ is the classical electron radius and

$\xi=\left[1-\frac{2\left(m_{e} c^{2}\right)^{2}}{E_{\gamma} E\left(1-\hat{e}_{\gamma} \cdot \hat{e}_{\mathrm{ph}}\right)}\right]^{1 / 2}$.

\subsection{Optical depth due to the companion starlight}

Starlight photons coming from the companion mid-A supergiant at a temperature $T \approx 8500 \mathrm{~K}[14,15]$ can cause absorption of gamma-rays. To see this clearly let us consider some simple geometry.

In Fig. 1 the observer is assumed to lay in the $x z$-plane and the gamma-ray path is described by the vector

$\vec{\rho}_{\gamma}=\rho_{\gamma}(\hat{x} \sin i+\hat{z} \cos i)=\rho_{\gamma} \hat{e}_{\gamma}$,

with $i=78^{\circ}$ as mentioned. The position of the star is given by $\vec{a}=a(\hat{x} \cos \phi+\hat{y} \sin \phi)$ and we suppose that the gammaray is produced in the jet at

$\vec{z}_{\mathrm{j}}=z_{\mathrm{j}}(\hat{x} \sin \theta \cos \psi+\hat{y} \sin \theta \sin \psi+\hat{z} \cos \theta)$,

where $\psi$ is the precessional phase. The position where the interaction with the soft photon takes place is indicated by

$\vec{r}_{\gamma}=\vec{z}_{\mathrm{j}}+\vec{\rho}_{\gamma}$ 
In this case, we set $\vec{l}_{\mathrm{ph}}=\left(\vec{r}_{\gamma}-\vec{a}\right)$ as the vector connecting the center of the star with the gamma-ray, and we call $\alpha$ the angle that it makes with $\hat{e}_{\gamma}$.

At each particular gamma-ray position, a convenient reference system can be chosen to perform the integration in the angles $\theta^{\prime}$ and $\phi^{\prime}$ in expression (1). This is done, as in Ref. [18], orientating the $z^{\prime}$-axis along the vector $\vec{l}_{\text {ph }}$. Accordingly, the angle $\phi^{\prime}$ varies between $\phi_{\min }^{\prime}=0$ and $\phi_{\max }^{\prime}=2 \pi$ and $\theta^{\prime}$ varies between $\theta_{\min }^{\prime}=0$ and $\theta_{\max }^{\prime}=$ $\arccos \left(\sqrt{1-\left(R_{\star} / l_{\mathrm{ph}}\right)^{2}}\right)$. Further orientating the $x^{\prime}$-axis so that the gamma-ray path is contained in the $x^{\prime} z^{\prime}$-plane, the gamma-ray direction can be written as $\hat{e}_{\gamma}=(\sin \alpha, 0, \cos \alpha)$ and hence, $\hat{e}_{\gamma} \cdot \hat{e}_{\mathrm{ph}}=\sin \alpha \sin \theta^{\prime} \cos \phi^{\prime}+\cos \alpha \cos \theta^{\prime}$. However, the dot-product involved for the evaluation of the cross section (2) and the minimum photon energy (10) is approximated by $\cos \alpha$, which does not depend on the primed angles. This last simplification that permits the analytic integration on $\phi^{\prime}$ and $\theta^{\prime}$, means only that in order to compute the cross section and the minimum energy, the starlight photons are all considered as being originated at the center of the star.

Integration in $\theta^{\prime}$ and $\phi^{\prime}$ of expression (1) thus gives

$$
\begin{aligned}
& \frac{d \tau_{\star}}{d E d \rho_{\gamma}}= 2 \pi n_{\star}(E) \sigma_{\gamma \gamma}\left(E, E_{\gamma}\right) \\
& \times\left[\cos \theta_{\min }^{\prime}-\cos \theta_{\max }^{\prime}\right. \\
&\left.-\frac{\cos \alpha}{2}\left(\sin ^{2} \theta_{\max }^{\prime}-\sin ^{2} \theta_{\min }^{\prime}\right)\right] .
\end{aligned}
$$

The density of radiation from the star can be approximated as usual by

$n_{\star}(E)=\frac{2 E^{2}}{(h c)^{3}\left(e^{E / k T_{\star}}-1\right)}\left(\mathrm{ph} \mathrm{cm}^{-3} \mathrm{erg}^{-1} \mathrm{sr}^{-1}\right)$.

Further integrating in target photon energy $E$ and along the gamma-ray path $\rho_{\gamma}$ yields the resulting starlight contribution to the optical depth

$\tau_{\star}=\int_{E_{\min }}^{\infty} d E \int_{0}^{\infty} d \rho_{\gamma} \frac{d \tau_{\star}}{d E d \rho_{\gamma}}$,

where

$E_{\min }=\frac{2\left(m_{e} c^{2}\right)^{2}}{E_{\gamma}\left(1-\hat{e}_{\gamma} \cdot \hat{e}_{\mathrm{ph}}\right)}$.

Once one relates the position vectors with time, one can obtain $\tau_{\star}\left(E_{\gamma}, t\right)$ as shown in Fig.2. These gamma-rays were assumed to be originated mostly near an injection point at the base of the approaching jet, $z_{0}=R_{0} / \tan \xi \simeq 1.3 \times$ $10^{9} \mathrm{~cm}$, where $\xi \simeq 0.6$ is the half opening angle of the jet (see Ref. [19]), and the initial jet radius is taken to be $R_{0}=$ $10 G M_{\mathrm{bh}} / c^{2}$. It can be seen from Fig.2 that this component of the opacity is notoriously more important when the star is in the foreground of the emission point, namely when it crosses the $x$-axis.

We note that to perform the intgral in Eq.(9), we have assumed for simplicity that the radiation density given by
Eq. (8) was artificially valid inside the star. However, as will be shown below, the absorption caused by $\gamma N$ interactions is by far the dominating one if the gamma-ray path directed to Earth has to travel through the star. This implies that, although the peaks in Fig. 2 could look different, an accurate expression for the radiation density inside the star will not affect the total optical depth.

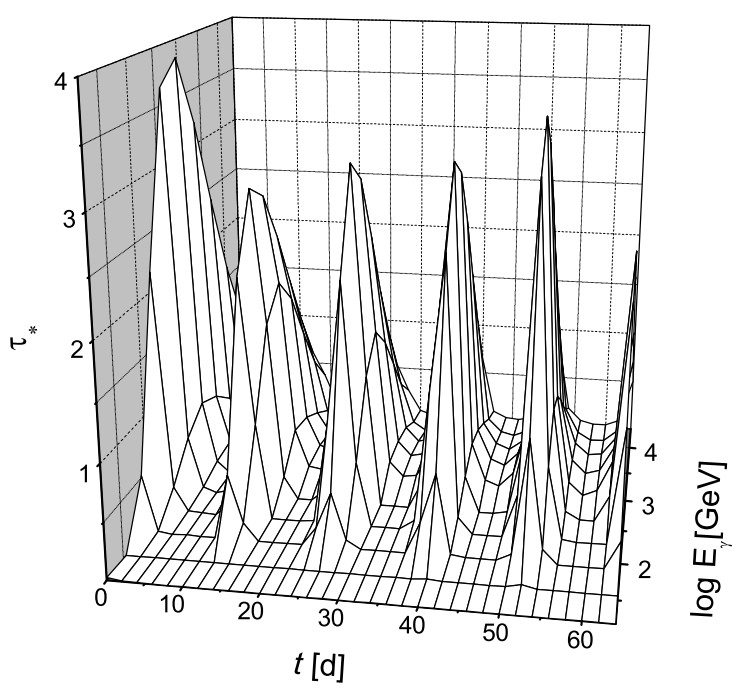

Fig. 2. Starlight optical depth.

\subsection{Optical depth due to the emission from the extended} disk

The extended disk wind is believed to be the origin of both the observed mid-IR and UV emission. In the first case, the reported free-free emission was detected in the range of wavelengths $2-12 \mu \mathrm{m}$ for which we adopt the following flux density fit [12],

$F_{\mathrm{ph}}=2.3 \times 10^{-23}\left(\frac{\lambda}{\mu \mathrm{m}}\right)^{-0.6}\left(\mathrm{erg} \mathrm{s}^{-1} \mathrm{~cm}^{-2} \mathrm{~Hz}^{-1}\right)$,

which corresponds an emitting region of radius $R_{\text {out }}=$ $50 R_{\odot}$. We need to estimate the radiation density of these mid-IR photons, i.e., the number of photons per unit volume, per unit energy per unit solid angle spanned by the emitting region. The corresponding mid-IR photon flux per unit frequency arriving at Earth can be written as

$\frac{d N_{\mathrm{ph}}}{d t d A d \nu}=\frac{F_{\mathrm{ph}}}{E}\left(\mathrm{ph} \mathrm{cm}^{-2} \mathrm{~s}^{-1} \mathrm{~Hz}^{-1}\right)$.

Considering that $d E / d \nu=h, d l_{\mathrm{ph}} / d t=c$, and that $d l_{\mathrm{ph}} d A=d V$, the radiation density coming from the whole emitting region is

$\frac{d N_{\mathrm{ph}}}{d V d E}=\frac{F_{\mathrm{ph}}}{E h c}\left(\mathrm{ph} \mathrm{cm}^{-3} \mathrm{erg}^{-1}\right)$.

Since the adopted fit for $F_{\mathrm{ph}}$ corresponds to the particular precessional phase $\psi_{\mathrm{IR}}=0.31 \times 2 \pi$ (see Ref. [12]), in that 
case, our line of sight makes an angle $i_{\mathrm{j}, \mathrm{IR}}$ with the normal to the disk midplane such that $\cos i_{\mathrm{j} . \mathrm{IR}}=\sin i \sin \theta \cos \psi_{\mathrm{IR}}+$ $\cos i \cos \theta$. Then, the area of the emitting region $\pi R_{\text {out }}^{2}$ spans a solid angle $\Delta \Omega=\left(\pi R_{\text {out }}^{2} \cos i_{\mathrm{j}, \mathrm{IR}}\right) / d^{2}$ as viewed from Earth, and we can estimate the corresponding radiation density as

$$
\begin{aligned}
n_{\mathrm{IR}} & \approx \frac{1}{\Delta \Omega}\left(\frac{d N_{\mathrm{ph}}}{d V d E}\right) \\
& =\frac{F_{\mathrm{ph}}}{E h c \pi \cos i_{\mathrm{j}, \mathrm{IR}}}\left(\frac{d}{R_{\text {out }}}\right)^{2}\left(\mathrm{ph} \mathrm{cm}^{-3} \mathrm{erg}^{-1} \mathrm{sr}^{-1}\right) .
\end{aligned}
$$

We proceed to integrate the mid-IR contribution of the optical depth $\tau_{\text {IR }}$ using the method described in Appendix A for a disk-like emitting zone. The inner radius of this zone is taken as the inner radius of the extended disk, $R_{\text {in }} \approx$ $2 G M_{\mathrm{bh}} / v_{w}^{2}$, as suggested in Ref. [20]. The obtained result is shown in Fig. 3 for gamma-rays originated at the base of the approaching jet.

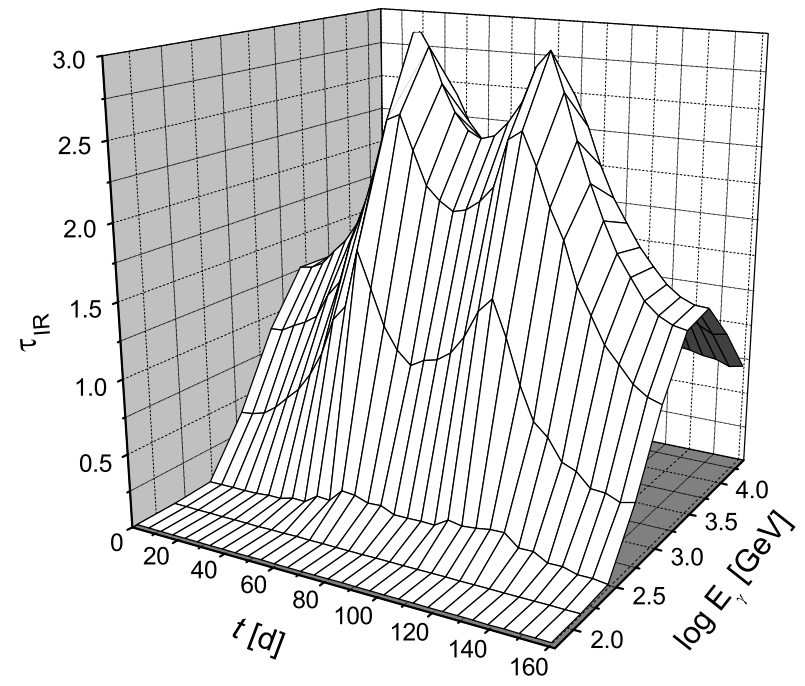

Fig. 3. Mid-IR optical depth as a function of time and gamma-ray energy for a gamma-ray produced at a height $z_{0}$ on the approaching jet.

As for the UV emission, it was detected in the range of wavelengths $\sim(1000-10000) \AA[11]$ and, as a first approach, we consider an associated blackbody temperature $T_{\mathrm{UV}}=21000 \mathrm{~K}$ for the disk in an edge-on state, with a corresponding radius of the emitting zone $R_{\text {out }}=33 R_{\odot}$. Therefore, we consider a radiation density for the UV emission as the one given in expression (8) with $T_{\star} \rightarrow T_{\mathrm{UV}}$.

The integration of this contribution to the total optical depth is also performed as it is described in Appendix A. The obtained contribution is shown in Fig. 4 as a function of time and gamma-ray energy.

It can be noted from this plot and from Fig. 3 that the maximum absorption occurs near the two times $t \sim 55 \mathrm{~d}$ and $t \sim 110 \mathrm{~d}$. Since the mid-IR and UV emissions are treated in this first analysis as being originated in the midplane of the extended disk, these times correspond to the cases when the gamma-rays originated at $z_{0}$ and directed to Earth run almost parallel to the mentioned emitting zone. This implies that $\gamma \gamma$ collisions will occur at larger angles resulting in higher cross sections and smaller threshold energies along a significant part of the gamma-ray path. At intermediate times, $55 \mathrm{~d}<t<110 \mathrm{~d}$, the mentioned gamma-rays have to pass through the midplane of the extended disk, and once they have done so, the collision angles are smaller, the threshold energy is larger, and hence the absorption is lower.

As for the dependence with the gamma-ray energy, it can be seen from Figs. 3 and 4 that the UV optical depth becomes important for $E_{\gamma} \gtrsim 50 \mathrm{GeV}$, while the mid-IR contribution will be significant for $E_{\gamma} \gtrsim 500 \mathrm{GeV}$.

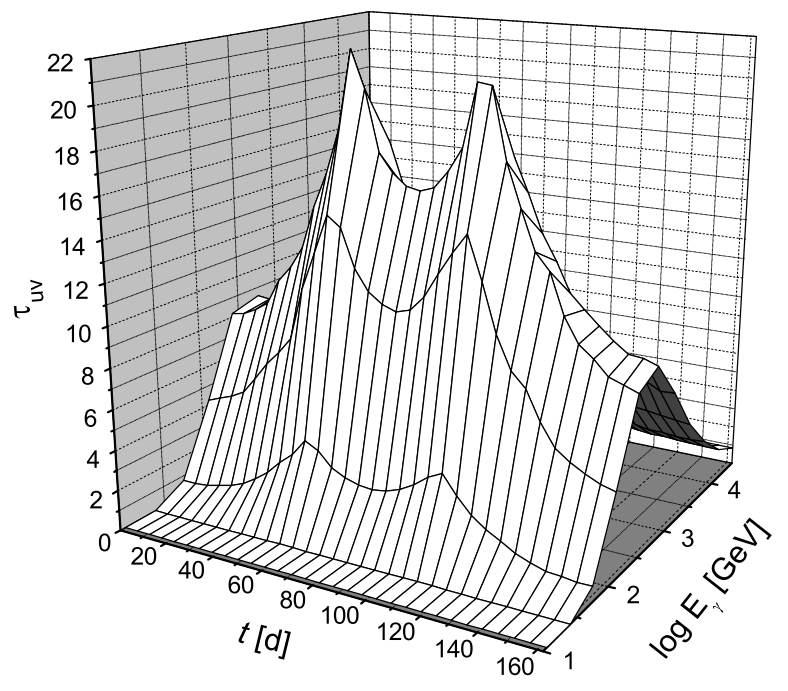

Fig. 4. UV optical depth as a function of time and gamma-ray energy for a gamma-ray produced at a height $z_{0}$ on the approaching jet.

\subsection{Optical depth due to $\gamma N$ interactions.}

The nucleons of the star and the thick extended disk can also absorb gamma-rays and photo-produce pions. We consider the cross section as in Ref. [21],

$\sigma_{\gamma N}=\left\{\begin{array}{cc}340 \mu \mathrm{b} & \text { for } 200 \mathrm{MeV}<E_{\gamma}<500 \mathrm{MeV} \\ 120 \mu \mathrm{b} & \text { for } E_{\gamma} \geq 500 \mathrm{MeV}\end{array}\right.$,

where the first case corresponds to the single pion channel and the second case to the multi-pion channel.

Considering the unit vector $\hat{Z}=(\sin \theta \cos \psi, \sin \theta \sin \psi, \cos \theta)$, which is perpendicular to the midplane of the disk wind, and the angle it makes with the gamma-ray position, $\theta_{Z}=\arccos \left(\hat{Z} \cdot \vec{r}_{\gamma} / r_{\gamma}\right)$, the density of the extended disk wind at the point $\vec{r}_{\gamma}$ is estimated to be

$\rho_{\mathrm{w}}\left(r_{\gamma}, \theta_{Z}\right)=\frac{\dot{M}_{\mathrm{w}}}{v_{\mathrm{w}} \Delta \Omega r_{\gamma}^{2}} \Theta\left[\theta_{Z}-\left(\frac{\pi}{2}-\alpha_{\mathrm{w}}\right)\right] \Theta\left[\left(\frac{\pi}{2}+\alpha_{\mathrm{w}}\right)-\theta\right.$ 女子 7.$)$ 
Here, $\Theta$ is the Heaviside step function and the solid angle element is related to the disk wind half opening angle $\alpha_{\mathrm{w}}$ by $\Delta \Omega=4 \pi \sin \alpha_{\mathrm{w}}$.

As for the star, we suppose that it has a density given by

$\rho_{\star}(r)=\frac{M_{\star}}{4 \pi R_{\star} r^{2}} \Theta\left(r-R_{\star}\right)$,

where $r$ represents the distance to the center of the star.

The optical depth due to $\gamma N$ interactions with the mentioned nucleons can be estimated as

$\tau_{\gamma N}\left(\vec{z}_{\mathrm{j}}\right)=\int_{0}^{\infty} d \rho_{\gamma} \sigma_{\gamma N} \frac{\left(\rho_{\star}+\rho_{\mathrm{w}}\right)}{m_{p}}$.

The obtained result is shown in Fig. 5 as a function of time for $200 \mathrm{MeV}<E_{\gamma}<500 \mathrm{MeV}$ and $E_{\gamma}>500 \mathrm{MeV}$. As it can be seen from this plot, periodic peaks of high absorption appear when the star is in the foreground. We note that the absorption caused by the disk wind is rather low. In particular, it vanishes when the approaching jet is most open to us (for $t \lesssim 12 \mathrm{~d}$ and $t \gtrsim 148 \mathrm{~d}$ ) because in those cases the gamma-ray path originated at the base of the jet never travels through the disk wind.

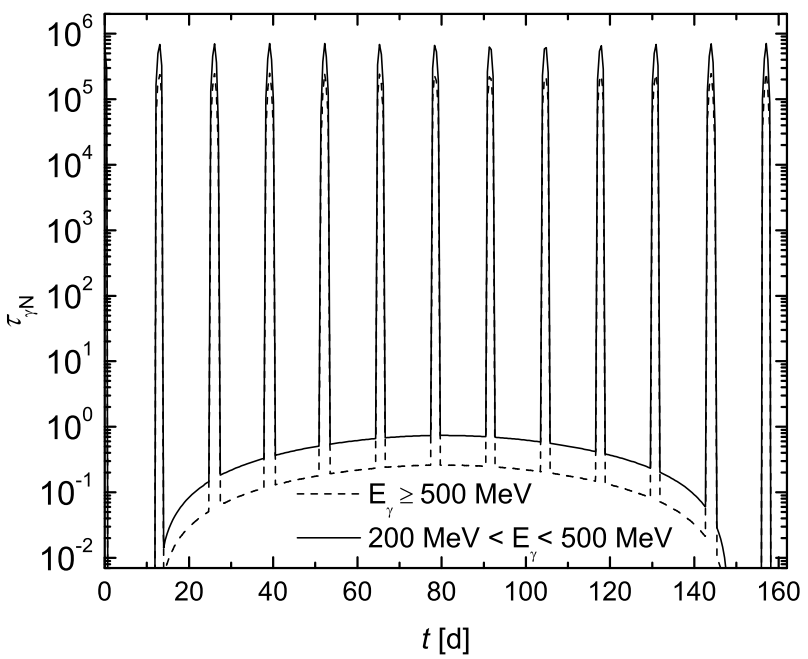

Fig. 5. $\gamma N$ optical depth as a function of time for $200 \mathrm{MeV}<E_{\gamma}<500 \mathrm{MeV}$ (solid line) and $E_{\gamma}>500 \mathrm{MeV}$ (dashed line). The sharp peaks are due to the orbital modulation.

\subsection{Total optical depth}

Analyzing the different contributions, it can be noted that below $E_{\gamma}=20 \mathrm{GeV}$, the only relevant source of absorption is due to the $\gamma N$ interactions with the nucleons of the extended disk and the star.

If we add up all the contributions calculated above for the case of gamma-rays originated at a height $z_{0}$ in the approaching jet, we can obtain the total optical depth which is shown in Fig. 6 as a function of time and gamma-ray energy. It can be seen clearly from this plot that the absorption caused by $\gamma N$ interactions with the nucleons of the star is important along all the range of gamma-ray energies studied $(200 \mathrm{MeV}-20 \mathrm{TeV})$.

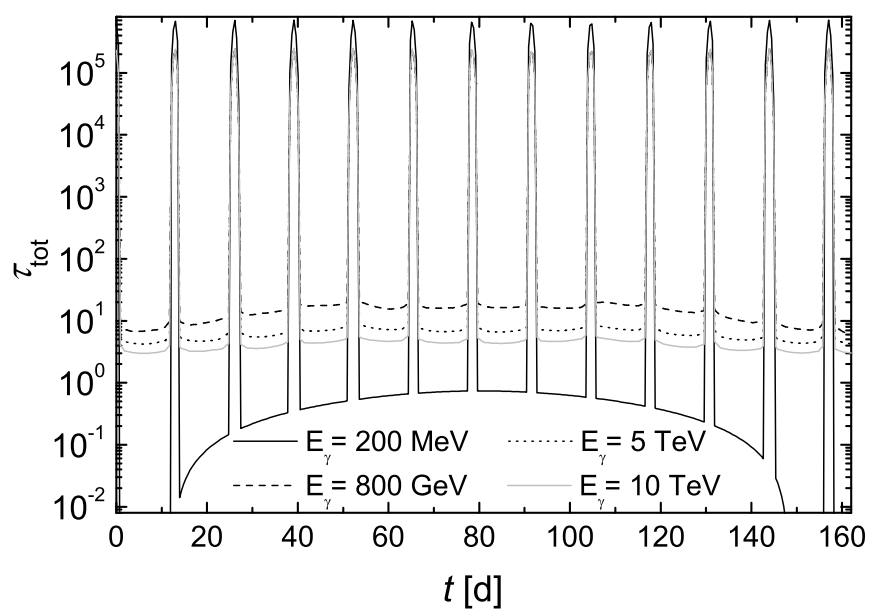

Fig. 6. Total optical depth for gamma-rays with as a function of time for $E_{\gamma}=200 \mathrm{MeV}$ (solid line), $E_{\gamma}=800 \mathrm{GeV}$ (dashed line), $E_{\gamma}=5$ $\mathrm{TeV}$ (dotted line), and $E_{\gamma}=10 \mathrm{TeV}$ (gray solid line).

\subsection{Dependence of the absorption on the gamma-ray injection point}

We shall now consider the cases where most of the gamma-rays are produced at a given height $z_{\mathrm{j}}>z_{0}$ along the approaching jet. The calculation of the various components of the optical depth can be performed as described in the previous section substituting $z_{0}$ by $z_{\mathrm{j}}$.

We show the obtained total optical depth as a function of $z_{\mathrm{j}}$ and the gamma-ray energy in Figs. 7 and 8 for $t \sim$ $162 \mathrm{~d}$ and $t \sim 81 \mathrm{~d}$ respectively, representing the cases when the absorption is maximum and minimum. Since at these times the star is not in the foreground, the optical depth is basically the one corresponding to the UV and mid-IR emission from the disk wind. At $t=162 \mathrm{~d}$, when the approaching jet is mostly open to our direction, the optical depth decreases with $z_{\mathrm{j}}$ because as $z_{\mathrm{j}}$ increases, the distance to be traveled by the gamma-ray directed to Earth over which $\gamma \gamma$ interactions are important decreases.

At $t=81 \mathrm{~d}$ there is an initial rise of the optical depth with $z_{\mathrm{j}}$. This is because at this particular time, the approaching jet as was referred here for simplicity, is actually not approaching the Earth but directed away from it. Then, as $z_{\mathrm{j}}$ increases, the distance along which $\gamma \gamma$ collisions are relevant increases for the gamma-rays coming in our direction. However, for still higher values, $z_{\mathrm{j}} \gtrsim 10^{11} \mathrm{~cm}$, the optical depth decreases with $z_{\mathrm{j}}$ since the density of UV radiation from the extended disk wind is lower.

\section{SIGNATURES ON A GAMMA-RAY SIGNAL}

In the present work we have not specified any particular gamma-ray emission process. Regardless of this, one possi- 


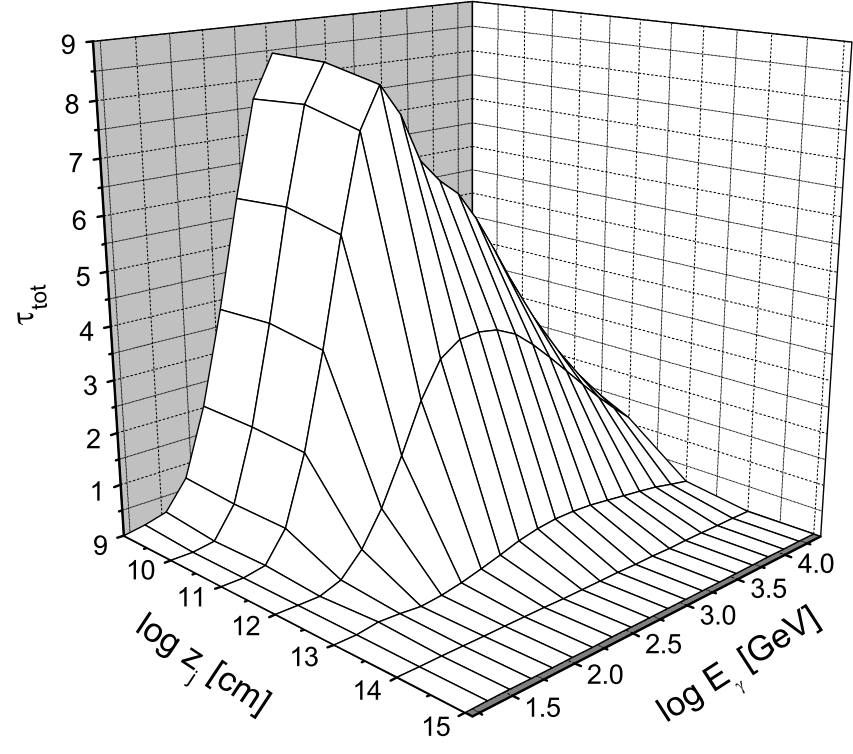

Fig. 7. Total optical depth at $t=162 \mathrm{~d}$ for gamma-rays originated with energy $E_{\gamma}$ at a distance $z_{\mathrm{j}}$ from the compact object along the approaching jet.

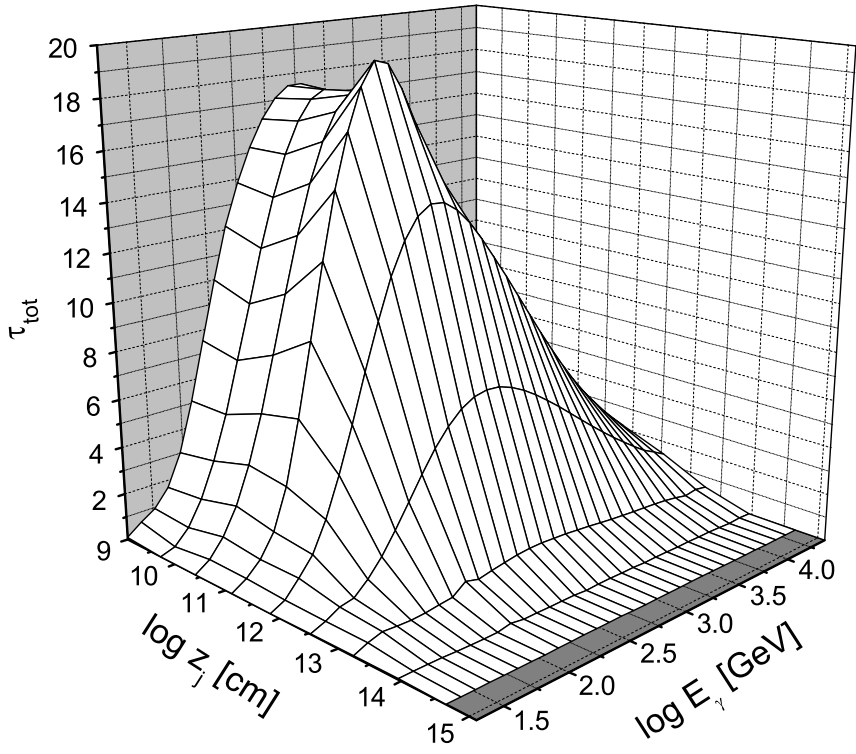

Fig. 8. Total optical depth at $t=81 \mathrm{~d}$ for gamma-rays originated with energy $E_{\gamma}$ at a distance $z_{\mathrm{j}}$ from the compact object along the approaching jet.

bility is that since the particle densities are expected to be highest in the inner jet, most of the gamma-rays could be produced at a short distance $z_{\mathrm{j}}$ from the compact object. In view of the recent detection of gamma-rays from similar systems, such as LS I +61 303, LS 5039, and Cyg X-1 $[4,5,6]$, and in an attempt to give an idea of the effect that the obtained optical depth may cause on an out-coming gamma-ray flux, we can assume that most of the radiation is produced near the base of the jets $\left(\right.$ at $z_{\mathrm{j}} \sim z_{0}$ ). In these conditions, if the spectrum of produced gamma-rays follows a power law as

$J_{\gamma}=K_{\gamma} E_{\gamma}^{-2}\left(\mathrm{ph} \mathrm{erg}^{-1} \mathrm{sr}^{-1} \mathrm{~cm}^{-2} \mathrm{~s}^{-1}\right)$,

where $K_{\gamma}$ is a constant, the total luminosity within an energy range $\left(E_{\gamma}^{\min }, E_{\gamma}^{\max }\right)$ is

$L_{\gamma}=\Delta A \int_{E_{\gamma}^{\min }}^{E_{\gamma}^{\max }} d E_{\gamma} E_{\gamma} J_{\gamma}\left(E_{\gamma}\right)$,

where $\Delta A$ is the element of area of the emitting region near the injection point. It follows, then, that

$K_{\gamma} \Delta A=\frac{L_{\gamma}}{\ln \frac{E_{\gamma}^{\max }}{E_{\gamma}^{\min }}}$,

which allows us to estimate the corresponding photon flux to be detected at the Earth as

$\Phi_{\gamma}(t)=\frac{\Delta A}{4 \pi d^{2}} \int_{E_{\gamma}^{\min }}^{E_{\gamma}^{\max }} d E_{\gamma} J_{\gamma}\left(E_{\gamma}\right) e^{-\tau_{\text {tot }}\left(t, E_{\gamma}\right)}$.

For illustration, we assume that the equivalent isotropic gamma-ray luminosity between $E_{\gamma}^{\min }=200 \mathrm{MeV}$ and $E_{\gamma}^{\max }=20 \mathrm{TeV}$ is $L_{\gamma} \approx 10^{36} \mathrm{erg} \mathrm{s}^{-1}$. The resulting flux is shown in the upper panel of Fig. 9, whereas the flux corresponding to energies $E_{\gamma}>800 \mathrm{GeV}$ is shown in the lower panel as compared to the upper limit given by HEGRA [22]. In the first case the obtained mean photon flux is

$$
\left.\left\langle\Phi_{\gamma}\right\rangle\right|_{E_{\gamma}>200 \mathrm{MeV}}=5 \times 10^{-8} \mathrm{ph} \mathrm{cm}^{-2} \mathrm{~s}^{-1},
$$

and in the second case,

$$
\left.\left\langle\Phi_{\gamma}\right\rangle\right|_{E_{\gamma}>800 \mathrm{GeV}}=4.7 \times 10^{-14} \mathrm{ph} \mathrm{cm}^{-2} \mathrm{~s}^{-1},
$$

which is below the HEGRA cut, $\Phi_{\gamma}^{\lim }=8.9 \times 10^{-13} \mathrm{ph} \mathrm{cm}^{-2} \mathrm{~s}^{-1}$.

We emphasize that these values are assumed only to make a qualitative description of a possible gamma-ray signal and that their true values may be obtained in a more detailed study regarding the emission process that could operate.

\section{DISCUSSION}

As it is reasonable to expect, the most noticeable absorption signature that can be imprinted on a gamma-ray flux from the inner regions of SS433 is given by the regular and dramatic absorption caused by the star when it crosses the line of sight. This effect takes place approximately once every 13 days during the time that the star blocks the emitting region $(\approx 2 \mathrm{~d})$. In practice, this could serve to determine the size of the companion star as well as the mass distribution of the stellar atmosphere as long 


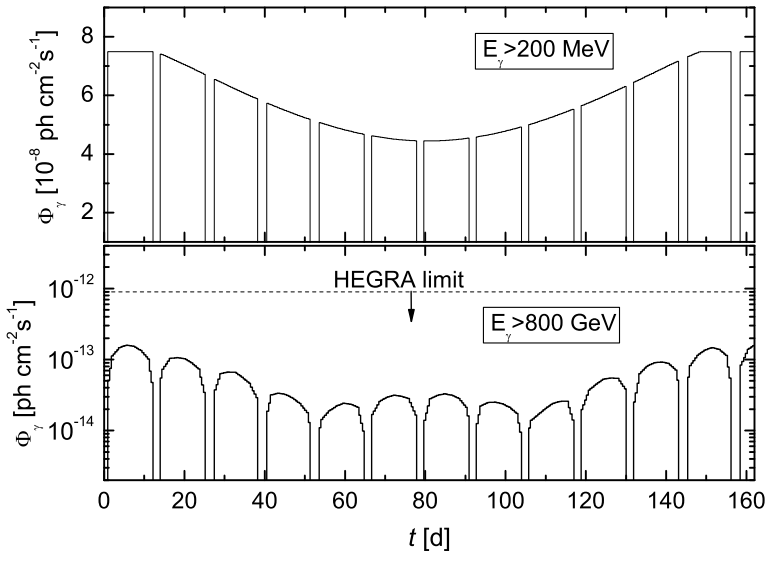

Fig. 9. Gamma-ray flux a function of time for $E_{\gamma}>200 \mathrm{MeV}$ (upper panel) and for $E_{\gamma}>800 \mathrm{GeV}$ (lower panel) as compared to the upper limit given by HEGRA. The assumed gamma-ray luminosity emitted is $L_{\gamma}=10^{36} \mathrm{erg} \mathrm{s}^{-1}$ and the maximum energy is $E_{\gamma}^{\max }=20 \mathrm{TeV}$

as sufficient time resolution can be achieved in the $\gamma$-ray lightcurves. It seems possible that, given the little absorption that corresponds to energies below $\sim 50 \mathrm{GeV}$ and the fact that more events are expected for lower energies, the GLAST instrument could detect a signal possibly with the time behavior predicted here. Another detectable absorption feature to observe at these energies corresponds to the $\gamma N$ interactions with the nucleons of the extended disk. This is expected to cause, because of the precession, a long term modulation of the signal reducing the maximum flux by a significant fraction as shown in the upper panel of Fig. 9 , provided that the bulk of the emission is produced near $z_{0}$.

As for higher energies, absorption through $\gamma \gamma$ interactions also becomes important. The dominant contribution is caused by the UV emission from the extended disk for $E_{\gamma} \gtrsim 50 \mathrm{GeV}$, while the mid-IR photons there originated can cause rather significant attenuation for $E_{\gamma} \gtrsim 500 \mathrm{GeV}$. The absorption due to the starlight photons is significant when the star is nearly in the foreground, almost eclipsing the black hole. This can be seen in the lower panel of Fig. 9, where the gamma-ray signal decreases and increases quite smoothly before and after the star has actually eclipsed the emission zone.

The fact that HEGRA has failed to detect anything greater than $\Phi_{\gamma}^{\lim }=8.9 \times 10^{-13} \mathrm{ph} \mathrm{s}^{-1} \mathrm{~cm}^{-2}$ for $E_{\gamma}>800$ $\mathrm{GeV}$, implies that maybe SS433 is not intrinsically luminous at these energies, or that gamma-rays could be actually produced at an inner injection point as illustrated here with a given luminosity, but nothing could be detected at the Earth because of absorption. If the assumed luminosity in gamma-rays is similar to the real intrinsic luminosity, a detection at these energies seems more difficult but not impossible with the forthcoming Cherenkov telescopes MAGIC II and VERITAS.

Finally we remark again that the conclusions drawn in the present study are independent of the gamma-ray pro- ducing mechanism. Taking into account reprocessing of gamma-rays due to cascading effects [23], would not either alter the absorption patterns here predicted. In case of an hadronic mechanism for gamma-ray production in the source (e.g. [24]), neutrino emission should be expected, as pointed out by several authors $[25,26]$. Contrary to gammarays, the neutrino signal is not affected by absorption, and its detection by instruments like IceCube might yield light on the relativistic particle content of the inner jets.

A comprehensive study, including the description of the physical mechanisms allowing the high-energy emission processes in SS433 will be presented elsewhere.

Acknowledgements We thank V. Bosch-Ramon for useful comments and an anonymous referee for constructive criticism. G.E.R. is supported by the Argentine agencies CONICET (PIP 5375) and ANPCyT (PICT 03- 13291 BID 1728/OC-AR). G.E.R. also acknowledges support from the Ministerio de Eduación y Ciencia (Spain) under grant AYA2007-68034-C03-01, FEDER funds. H.R.C. is supported by CNPq and FUNCAP, Brazil, and M.M.R. is supported by CONICET, Argentina. M.M.R. is also grateful to O. A. Sampayo for useful discussions relevant to the present work.

\section{References}

[1] S. Fabrika, Astrophysics and Space Physics Reviews, 12, 1 (2004)

[2] E. Gallo et al. Nature 436, 819 (2005)

[3] G. M. Dubner, M. Holdaway, W. M. Goss, I. F. Mirabel, Astron. J. 116, 1842 (1998)

[4] J. Albert et al. (MAGIC coll.), Science, 312, 1771 (2006)

[5] F. A. Aharonian et al. Science 309, 746 (2005)

[6] J. Albert et al. (MAGIC coll.), Astrophys. J. Lett., 665, L51 (2007)

[7] G. E. Romero, H. R. Christiansen, M. Orellana, Astrophys. J. 632, 1093 (2005)

[8] G. E. Romero, A. Okazaki, M. Orellana, S.P. Owocki, Astron. Astrophys., 474, 15 (2007)

[9] M. Böttcher, Astropart. Phys. 27, 287 (2007)

[10] T. Zwitter, M. Calvani, S. D'Odorico, Astron. Astrophys., 251, $92(1991)$

[11] D. R. Gies, M. V. McSwain, R. L. Riddle, Z. Wang, P. J. Wiita, D. W. Wingert, Astrophys. J. 566, 1069 (2002)

[12] Y. Fuchs, L. K. Miramond, P. Ábrahám, Astron. Astrophys. 445, 1041 (2006)

[13] A. M. Cherepashchuk et al., Astron. Astrophys. 437, 561 (2005)

[14] T. C. Hillwig et al., Astrophys. J. 615, 422 (2004)

[15] A. D. Barnes et al., Astron. Astrophys. 365, 296 (2006)

[16] P. P. Eggleton, Astrophys. J. 268, 368 (1983)

[17] R. J. Gould, G. P. Schréder, Phys. Rev., 155, 1404 (1967)

[18] G. Dubus, Astron. Astrophys. 451, 9 (2006)

[19] H. L. Marshall, C. R. Canizares, N. S. Schulz, Astrophys. J. 564, $941(2002)$

[20] M. C. Begelman, A. R. King, J. E. Pringle, MNRAS 370, 399 (2006)

[21] A. M. Atoyan, C. D. Dermer, Astrophys. J. 586, 79 (2003)

[22] F. Aharonian et al., Astron. Astrophys. 439, 635 (2005)

[23] W. Bednarek, MNRAS 371, 1737 (2006)

[24] G. E. Romero, D. F. Torres, M. M. Kaufman-Bernadó, I. F. Mirabel, Astron. Astrophys. 410, L1 (2003) 
[25] H. R. Christiansen, M. Orellana, G. E. Romero, Phys. Rev. D, 73, 063012 (2006)

[26] F. A. Aharonian, L. A. Anchordoqui, D. Khangulyan, T. Montaruli, J. Phys. Conf. Ser., 39, 408 (2006)

[27] P. A. Becker, M. Kafatos, Astrophys. J. 453, 83 (1995)

[28] V. Barger, R. Phillips, Collider Physics, Addison-Wesley (California, Redwood City, 1987), p. 397

\section{Appendix A. Calculation of the gamma-ray absorption near a disk-like emitting region}

We consider the absorption of gamma-rays as they travel near a disk that emits soft photons. This disk, with an inner radius $R_{\text {in }}$ and an outer radius $R_{\text {out }}$, lies in a plane perpendicular to the jets, where the gamma-rays emerge. For gamma-rays produced with energy $E_{\gamma}$, traveling in a direction $\hat{e}_{\gamma}$, the differential optical depth due to photons with a radiation density $n_{\mathrm{ph}}$ and a direction given by $\hat{e}_{\mathrm{ph}}$, can be written as (e.g [27])

$d \tau_{\gamma \gamma}=\left(1-\hat{e}_{\mathrm{ph}} \cdot \hat{e}_{\gamma}\right) n_{\mathrm{ph}} \sigma_{\gamma \gamma} \frac{\cos \eta R_{\mathrm{d}} d R_{\mathrm{d}} d \phi_{\mathrm{d}}}{l_{\mathrm{ph}}^{2}} d \rho_{\gamma} d E$.

Here the $R_{\mathrm{d}}$ is the length of the vector $\vec{R}_{\mathrm{d}}$ signaling a point in the disk, $\phi_{\mathrm{d}}$ is its corresponding azimuthal angle, and $l_{\mathrm{ph}}$ is the magnitude of the vector $\vec{l}_{\mathrm{ph}}$ connecting the point on the disk to the gamma-ray position. This vector makes an angle $\eta$ with the normal of the disk plane. All the angles and vectors involved in (A.1) are then to be expressed in a new coordinate system with its $Z$-axis oriented along the axis of the approaching jet.

It is worth noting that the radiation density $n_{\text {ph }}$ appearing in expression (A.1) represents the number of soft photons per unit volume, per unit energy, per unit solid angle. Hence, for photons originated on a differential area element $d a=R_{\mathrm{d}} d R_{\mathrm{d}} d \phi_{\mathrm{d}}$ on the disk plane, the differential solid angle spanned is $d \Omega=(d a \cos \eta) / l_{\mathrm{ph}}^{2}$, which appears in expression (A.1).

In the system fixed to the compact object the unit vector $\hat{e}_{\gamma}$ is given by Eq. (4) in terms of the unit vectors $\hat{x}, \hat{y}$ and $\hat{z}$, and the basis of the new system can be obtained by transforming them according to

$R=\left(\begin{array}{ccc}\cos \psi \cos \theta & -\sin \psi & \cos \psi \sin \theta \\ \sin \psi \cos \theta & \cos \psi & \sin \psi \sin \theta \\ -\sin \theta & 0 & \cos \theta\end{array}\right)$

Therefore, the unit vectors of the new coordinate system can be expressed in terms of fixed basis as

$\hat{X}=\hat{x} \cos \theta \cos \psi+\hat{y} \cos \theta \sin \psi-\hat{z} \sin \theta$

$\hat{Y}=-\hat{x} \sin \psi+\hat{y} \cos \psi$

$\hat{Z}=\hat{x} \sin \theta \cos \psi+\hat{y} \sin \theta \sin \psi+\hat{z} \cos \theta$,

and the unit vector $\hat{e}_{\gamma}$ can be written as

$\hat{e}_{\gamma}=\hat{X}\left(\hat{e}_{\gamma} \cdot \hat{X}\right)+\hat{Y}\left(\hat{e}_{\gamma} \cdot \hat{Y}\right)+\hat{Z}\left(\hat{e}_{\gamma} \cdot \hat{Z}\right)$

$$
\begin{aligned}
\hat{e}_{\gamma} & =\hat{X}(\cos \theta \sin \psi \sin i-\sin \theta \cos i) \\
& -\hat{Y}(\sin \psi \sin i) \\
& +\hat{Z}(\sin \theta \cos \psi \sin i+\cos \theta \cos i) .
\end{aligned}
$$

Since the position of the gamma-ray is

$\vec{r}_{\gamma}=\hat{e}_{\gamma} \rho_{\gamma}+\hat{Z} z_{\mathrm{j}}$

and the position on the disk is

$\vec{R}_{\mathrm{d}}=R_{\mathrm{d}}\left(\hat{X} \cos \phi_{\mathrm{d}}+\hat{Y} \sin \phi_{\mathrm{d}}\right)$,

the vector $\vec{l}_{\mathrm{ph}}$ can be obtained as $\vec{l}_{\mathrm{ph}}=\vec{r}_{\gamma}-\vec{R}_{\mathrm{d}}$.

The resulting optical depth is given by the quadruple integral

$\tau_{\gamma \gamma}=\int_{0}^{\infty} d \rho_{\gamma} \int_{0}^{2 \pi} d \phi_{\mathrm{d}} \int_{R_{\mathrm{in}}}^{R_{\text {out }}} d R_{\mathrm{d}} \int_{E_{\min }}^{E_{\max }} d E \frac{d \tau_{\gamma \gamma}}{d \rho_{\gamma} d \phi_{\mathrm{d}} d R_{\mathrm{d}} d E}$,

where

$E_{\min }=\frac{2\left(m_{e} c^{2}\right)^{2}}{E_{\gamma}\left(1-\hat{e}_{\gamma} \cdot \hat{e}_{\mathrm{ph}}\right)}$.

The integral can be performed using a Monte Carlo method, that is, introducing the variables $x_{\rho}, x_{\phi}, x_{R}$, and $x_{E}$ as

$$
\begin{aligned}
\rho_{\gamma} & =\rho_{1} x_{\rho} \\
\phi_{\mathrm{d}} & =2 \pi x_{\phi} \\
R_{\mathrm{d}} & =R_{\text {in }}+\left(R_{\text {out }}-R_{\text {in }}\right) x_{R} \\
E & =E_{\text {min }}+\left(E_{\text {max }}-E_{\text {min }}\right) x_{E},
\end{aligned}
$$

then the integral (A.8) can be written as

$\tau_{\gamma \gamma}=\int_{0}^{1} d x_{\rho} \int_{0}^{1} d x_{\phi} \int_{0}^{1} d x_{R} \int_{0}^{1} d x_{E} f\left(x_{\rho}, x_{\phi}, x_{R}, x_{E}\right)$,

where

$f\left(x_{\rho}, x_{\phi}, x_{R}, x_{E}\right)=2 \pi \rho_{1}\left(R_{\text {out }}-R_{\text {in }}\right) \times$

$$
\left(E_{\max }-E_{\min }\right) \frac{d \tau_{\gamma \gamma}}{d \rho_{\gamma} d \phi_{\mathrm{d}} d R_{\mathrm{d}} d E}
$$

Here the upper limit of the integration in $\rho_{\gamma}$ is taken as $\rho_{1} \approx 10 R_{\text {out }}$ where the integrand is significative.

The method relies on the fact that, by the Mean-Value Theorem, the integral can be approximated by

$\tau_{\gamma \gamma} \approx \frac{1}{N} \sum_{i=1}^{N} f\left(x_{\rho}(i), x_{\phi}(i), x_{R}(i), x_{E}(i)\right)$,

where each variable takes a random number between 0 and 1. The right hand side is then the statistical average of $N$ evaluations of the function $f$. The error goes like $1 / \sqrt{N}$, which in this case of four integration variables makes it a quite accurate method as compared to the iterative ones (see e.g. [28]). 